\title{
Lernen im Kontext von Biografie und Lebenslauf
}

\author{
Jochen Kade $\cdot$ Sigrid Nolda
}

Online publiziert: 11. März 2015

(C) Die Autor(en) 2015. Dieser Artikel ist auf Springerlink.com mit Open Access verfügbar

Spätestens seit der 1986 von Hans Tietgens in seiner Studie „Erwachsenenbildung als Suchbewegung“ diagnostizierten fundamentalen Begründung der Erwachsenenbildung aus der Biografie der Erwachsenen ist die Biografieforschung eine der relevanten Forschungszweige zum Lehren und Lernen und damit zur Bildung Erwachsener. Ihre Diskussion begleitet alle Etappen dieser Zeitschrift: angefangen mit Peter Alheits Artikel über die Biographieforschung in der Erwachsenenbildung (1984) über die Themenhefte Biographieforschung und biographisches Lernen (1996) und Biographie und Bildung (2008) bis zu dem vorliegenden Heft der nunmehr unter dem erziehungswissenschaftlich programmatischen Namen stehenden Zeitschrift für Weiterbildungsforschung. Abzulesen sind bereits an den Titeln dieser Schwerpunkte wesentliche Entwicklungslinien im Verhältnis von Biografie und Erwachsenenbildung. Es kennzeichnet die zunehmende Verwissenschaftlichung der Erwachsenen-/ Weiterbildung und der Reflexion über sie, dass die Biografieforschung schrittweise stärker auf die Erwachsenen-/Weiterbildung als einen disziplinären Zusammenhang von Theorieentwicklung und Feldforschung bezogen wird.

Die Biografieforschung thematisiert inzwischen nicht nur den Prozess des Individuums, das sich unter den Bedingungen moderner Gesellschaften im gesamten Verlauf seines Lebens bildet und - fokussierter dann - den einzelnen Erwachsenen als Teilnehmenden an Veranstaltungen der organisierten Erwachsenenbildung. Sie hat inzwischen auch die Felder von Profession/Professionalität einerseits und Organisa-

Prof. Dr. J. Kade $(\bowtie)$

Goethe-Universität Frankfurt am Main, Frankfurt am Main, Deutschland

E-Mail:kade@em.uni-frankfurt.de

Univ.-Prof. Dr. S. Nolda

Technische Universität Dortmund, Dortmund, Deutschland

E-Mail: Sigrid.Nolda@fk12.tu-dortmund.de 
tion/Institution erschlossen. Die Biografieforschung ist zu einer zentralen Perspektive und auch zu einem Instrument bei der Erschließung und dann auch kontinuierlichen Untersuchung neuer disziplinärer Schwerpunkte und gesellschaftlicher Erscheinungen in ihrer Bedeutung für die Erwachsenenbildung geworden.

In den 1980er Jahren waren es noch vorwiegend Theorien und Konzepte psychologischer und soziologischer Lebenslaufforschung, die auf die Erwachsenenbildung angewendet wurden. Inzwischen sind aus der erziehungswissenschaftlichen, auch oder ausschließlich auf Erwachsene gerichteten, mittlerweile auch international ausgerichteten Biografieforschung selbst empirisch fundierte Konzepte entwickelt worden, die den erziehungswissenschaftlichen Status dieser Forschungsrichtung befestigt haben. Zugleich haben sich zunehmend elaboriertere Diskurse zu methodologischen und methodisch relevanten Fragen etabliert. So werden etwa qualitativ ausgerichtete Längsschnittstudien, die in den 1980 Jahren noch nicht auf dem inzwischen entwickelten Niveau möglich waren, als Ausgangspunkt von neuen Fragestellungen und als Korrektur bisher gültiger Annahmen über lebenslauf- und biografierelevante Lernpraktiken Erwachsener genutzt. Diese Diskurse haben auch das Konzept der Biografie und spezieller der Bildungsbiografie - in grundlagentheoretischer Absicht - zum Thema gemacht. So wird etwa das Zeitdefizit der erziehungswissenschaftlichen Biografieforschung analysiert und die (Bildungs-)Biografie selbst als verzeitlicht und damit als Moment in einem den Lebenslauf diskontinuierlich begleitenden Prozess des Biografisierens ausgewiesen. Indem die ursprünglich als Gegenkonzept zum Lebenslauf verstandene Biografie mit diesem relationiert und als ein soziales Ereignis im Lebenslauf akzentuiert wird, kann auch die Gesellschaftlichkeit und Historizität von (Bildungs-)Biografien empirisch tiefer analysiert werden. Auf der Grundlage dieses erweiterten kategorialen Rahmens ist ein facettenreiches Spektrum theoretisch elaborierter und methodisch anspruchsvoller Forschung zur Analyse von Lehr-, Lern- und Bildungsprozessen im Lichte von Fragestellungen der Biografie- und Lebenslaufforschung entstanden.

Das Thema dieses Heftes referiert auf diese neuere Theorie- und Forschungslage der Biografieforschung im Kontext der Erwachsenen-/Weiterbildung. Es ist theoretisch und empirisch gleichermaßen akzentuiert. Vor dem Hintergrund erziehungswissenschaftlich orientierter Diskurse wird der Schwerpunkt dabei auf die Verknüpfung von Lebenslauf und Biografie im Zusammenhang von Lern- und Bildungsprozessen Erwachsener innerhalb und außerhalb von Institutionen gelegt, und damit auf Lernen und Bildung in der ganzen Breite und Tiefe des Lebenslaufs.

Den Beiträgen vorangestellt ist ein Überblick von Cornelia Maier-Gutheil, in dem der aktuelle Stand der Entwicklung auch der internationalen lebenslaufbezogenen und bildungsbiografieorientierten Forschung zu Lern- und Bildungsprozessen Erwachsener dargestellt wird. Er dient der Verortung der folgenden Beiträge.

In den Beiträgen von Ines Himmelbach, Sascha Benedetti sowie von Sigrid Nolda und Jochen Kade wird die Bereicherung lebenslaufbezogener Biografieforschung durch längsschnittliche Interviewstudien am Beispiel der Lebensphase des Alterns, des freiwilligen Engagements Erwachsener und der Praktik des Fremdsprachenlernens dargestellt. Im Rahmen einer querschnittlichen Interviewstudie analysieren Helza Ricarte Lanz und Juliane Noack Napoles die Motive von Frauen, die sich entschieden haben, in der Kindertagespflege tätig sein zu wollen. 
Eine diskurssensible, stärker theoretische Ausrichtung wird von Daniela Rothe und Astrid Seltrecht vorgenommen. Von Daniela Rothe wird auf die unterschiedlichen Konstruktionen des lernenden Subjekts in der programmatischen Bildungspolitik, der empiriegestützten Biografieforschung und in den didaktischen Modellen biografieorientierter Bildungsarbeit aufmerksam gemacht. Von Astrid Seltrecht wird, den Thementeil abschließend, das Verlernen zum Thema gemacht. In Abhebung von dessen alltagsweltlichem Verständnis wird ein erziehungswissenschaftlichen Begriff des Verlernens erörtert und vor diesem Hintergrund eine Heuristik zu dessen empirischer Analyse vorbereitet.

Im Forum dieses Heftes, in dem nicht zum Schwerpunkt gehörende Beiträge veröffentlicht werden, präsentieren Susanne Wißhack und Sabine Hochholdinger eine Analyse von Train-the-Trainer-Seminaren aus einer professionstheoretischen Perspektive. Aus der Analyse werden Potenziale für eine stärkere Standardisierung der Trainerausbildung für die betriebliche und berufliche Weiterbildung abgeleitet.

Im Forum des kommenden Heftes wird - so viel kann vorweggenommen werden - der Empirie und Theorie, Wissen und Können, Lernen und Bildung umgreifende Diskurs zu Biografie und Lebenlauf aus diesem Heft noch einmal fortgeführt, u. a. mit einem in Vorbereitung befindlichen Beitrag von Burkhard Schäffer, Olaf Dörner und Franz Krämer über die Bedeutung des Konzepts der Lebensphasen für die Biografieforschung.

Open Access Dieser Artikel unterliegt den Bedingungen der Creative Commons Attribution License. Dadurch sind die Nutzung, Verteilung, und Reproduktion erlaubt, sofern der/die Originalautor/en und die Quelle angegeben sind.

\section{Literatur}

Kade, J., Nolda, S. (2012). Qualitative Forschungskulturen zum Lernen Erwachsenes. In: Schäffer, B., Dörner, O. (Hrsg.). Handbuch Qualitative Erwachsenen- und Weiterbildungsforschung (S. 641-655). Opladen: Barbara Budrich. 